\title{
Percepción del paciente hospitalizado sobre el cuidado brindado por estudiantes de enfermería
}

\author{
Inpatient perception of the care provided by nursing students \\ Sandra Milena Campiño-Valderrama' orcid.org/0000-0002-8754-4777 \\ Paula Andrea Duque ${ }^{* *}$ orcid.org/0000-0001-7237-6195 \\ Víctor Hugo Cardozo' orcid.org/0000-0003-2482-0938
}

1 Programa de Enfermería, Universidad Católica de Manizales. Manizales, Colombia

Fecha de recepción: Julio 25 - 2017

Fecha de revisión: Julio 5 - 2018

Fecha de aceptación: Agosto 9 - 2019

Campiño-Valderrama SM, Duque PA, Cardozo VH. Percepción del paciente hospitalizado sobre el cuidado brindado por estudiantes de enfermería. Univ. Salud. 2019;21(3):215-225. DOI: http://dx.doi.org/10.22267/rus.192103.158

\section{Resumen}

Introducción: La enfermería ha evolucionado su quehacer en torno a los constructos teóricos que la componen, éste crecimiento progresivo incluye el desarrollo de procesos de enseñanza resignificados en los futuros profesionales, que deben ser validados en torno a las respuestas humanas en la relación enfermero - paciente. Objetivo: Describir la percepción del paciente hospitalizado sobre el cuidado brindado por estudiantes de enfermería. Materiales y métodos: Estudio cuantitativo, descriptivo, de corte trasversal, realizado con 356 pacientes que recibieron cuidado por parte de estudiantes de enfermería durante en instituciones de salud, se aplicó el instrumento Percepción del Cuidado Humanizado de enfermería versión 3. Se analizó con estadística descriptiva y prueba no paramétrica mediante probabilidad Kruskal-Wallis. Resultados: Los pacientes manifestaron que "siempre se percibe un cuidado humanizado", en cuanto a las preguntas relacionadas con instrucciones sobre el autocuidado, identificación de necesidades de tipo físico, psicológico y espiritual se obtuvieron puntajes bajos. Conclusión: Es necesario abordar el tema del cuidado humanizado como fenómeno de interés de la disciplina al identificar fortalezas y debilidades con estudios de pertinencia en torno al cuidado.

Palabras clave: Percepción; estudiantes de enfermería; cuidado de enfermería; humanización de la atención. (Fuente: DeCS, Bireme).

\begin{abstract}
Introduction: Nursing has evolved its work around the theoretical constructs that compose it, this progressive growth includes the development of resignified teaching processes for future professionals, which must be validated around human responses in the nurse - patient relationship. Objective: Describe the perception of the hospitalized patient about the care provided by nursing students. Materials and methods: A quantitative, descriptive, cross-sectional study, conducted with 356 patients who received care from nursing students within health institutions. The Perception of Humanized Nursing Care instrument - version 3 was applied. It was analyzed with descriptive statistics and a non-parametric test using Kruskal-Wallis probability. Results: The patients stated that "humanized care is always perceived", regarding the questions related to instructions on self-care; low scores were obtained in identification of physical, psychological and spiritual needs. Conclusion: It is necessary to address the issue of humanized care as a phenomenon of interest of the discipline when identifying strengths and weaknesses with studies of relevance around care.
\end{abstract}

Key words: Perception; nursing students; nursing care; humanization of attention. (Source: DeCS, Bireme).

\footnotetext{
*Autor de correspondencia

Paula Andrea Duque

e-mail: paduque@ucm.edu.co
} 


\section{Introducción}

La profesión de enfermería es definida como una disciplina y una ciencia en construcción, que tiene como núcleo fundamental, estudiar la experiencias de cuidado que vivencian los seres humanos, ya sea en proceso de salud o enfermedad durante el desarrollo del ciclo vital(1); como disciplina profesional, la enfermería ha evolucionado su quehacer en torno a los constructos teóricos que la componen, éste crecimiento progresivo incluye el desarrollo de procesos de enseñanza que deben ser validados en torno a las respuestas humanas. La interacción entre el futuro profesional de enfermería y el sujeto de cuidado es un proceso que se construye en un momento de atención para mantener el confort del paciente y sus familiares(2), que por consiguiente le da sentido al acto cuidar, el cual se estructura desde los ámbitos históricos, culturales, sociales y personales del momento en que acontece.

El cuidado como acción fundamental de enfermería, ha evolucionado dentro su desarrollo histórico, constituyéndose en un proceso ordenado y objetivo que se sustenta en el método científico y que busca el equilibrio de la persona, de acuerdo a las acciones derivadas del proceso enfermero; dentro de los procesos formativos se hace imperativa la promoción de un liderazgo de enfermería que trascienda de lo individual y lo social, al considerar las necesidades, los deseos y las creencias de las personas; sin embargo, el quehacer profesional de enfermería en mucha ocasiones se visualiza como una atención que carece de contacto directo con la persona que se cuida, limitándose a la curación de la enfermedad y a la atención instrumental del paciente, olvidando que en cualquier escenario en donde se encuentre, se debe procurar girar en torno las respuestas $\mathrm{y}$ necesidades humanas(3).

El cuidado humanizado es la esencia fundamental de enfermería que se encarga del acompañamiento, la escucha, de brindar un trato digno basado en conocimientos científicos y al mismo tiempo éticos y morales para poder proporcionar un estado de confort y recuperación al usuario(4). En enfermería el cuidado se ha convertido en el centro de la praxis y un aspecto central debe ser valorar que estos cuidados se brinden en forma humanizada, para beneficio de los usuarios y de los profesionales de enfermería; por lo tanto, mejorar la calidad de los cuidados en los servicios de salud es fundamental para mejorar la práctica en el actual modelo de cuidado para la salud(5).

La labor del docente de enfermería, dentro del acto educativo, requiere la consolidación del cuidado con una pedagogía activa y desde la alteridad que se requiere para influir en el estudiante, de tal manera que se reconozca en el sujeto de cuidado la verdadera esencia del ámbito disciplinar y que posteriormente lo haga visible en su actuar como profesional de la salud. Ante el alto riesgo de deshumanización en el cuidado del paciente, a causa de la reestructuración administrativa de la mayoría de los sistemas del cuidado de la salud en el mundo, se hace necesario el rescate del aspecto humano, espiritual y transpersonal en la práctica clínica, administrativa, educativa y de investigación por parte de los profesionales de enfermería, demandando una revisión y seguimiento permanente a los currículos que lleven al estudiante a enfrentar los fenómenos del cuidado desde la realidad de los seres humanos y del más íntimo momento que se propicia en la relación enfermero - paciente, donde lo realmente importante es conocer que piensa y siente la persona a quien se le brinda el cuidado(6).

Cuidar humanamente implica una asistencia de calidad, llevando beneficios tanto para los profesionales de enfermería y el equipo multidisciplinar, ya que proporciona un ambiente de trabajo ético y agradable para el paciente enfermo, con necesidades, sentimientos, dificultades personales, que pasa a ser el centro de atención, siendo cuidado de forma holística e integral(7). La teoría de Jean Watson se basa en diez procesos caritas o proceso de cuidar, sustentados en aspectos filosóficos que permiten comprender la praxis o "momento de cuidados", cada uno de ellos sustenta la manera de ofrecer un cuidado desde la comprensión y el establecimiento de una adecuada relación interpersonal. Específicamente en el proceso caritas número seis, Watson hace un llamado al uso sistemático del método de resolución de problemas para la toma de decisiones, atributo íntimamente relacionado con la motivación por la superación constante; cualidad que cuando está presente se expresa en el deseo de estudiar y adquirir nuevos conocimientos para perfeccionar la práctica diaria ${ }^{(8)}$.

Esto plantea la necesidad de gestar procesos investigativos que proporcionen una mirada real del futuro profesional y las escuelas de formación para 
determinar los resultados de sus planes de cuidado y la atención con calidad al paciente. Es necesario comprender la necesidad de involucrar a los estudiantes en el logro de competencias para brindar un cuidado humanizado soportado por sólidos conocimientos teóricos que se incorporen con habilidad, empatía, vocación, paciencia y respeto por la dignidad de la persona(7). Es por esto que el presente estudio tuvo como objetivo describir la percepción del paciente hospitalizado sobre el cuidado brindado por estudiantes de enfermería de la Universidad Católica de Manizales - Colombia, teniendo como referente investigativo el modelo de cuidado humanizado de Jean Watson.

\section{Materiales y métodos}

\section{Tipo de estudio y diseño}

Se realizó un estudio cuantitativo descriptivo y prospectivo de corte transversal, durante el año 2016, para medir la percepción que tiene el paciente, sobre en cuidado humanizado brindado por los estudiantes de enfermería de la Universidad Católica de Manizales. Durante este tiempo los estudiantes de tercero a sexto semestre realizaron sus prácticas clínicas por las áreas en que se encontraban de acuerdo al semestre, en diferentes escenarios hospitalarios de la ciudad de Manizales, bajo el acompañamiento de un docente de manera permanente, en el marco de la relación docenciaservicio; se tuvo en cuenta el tiempo en que el estudiante estuvo al cuidado del paciente que le fuese asignado, con el fin de medir adecuadamente la percepción del cuidado.

\section{Población y muestra}

Para la selección de la muestra fue necesario tomar un universo no conocido y esto hace referencia a que no existe un número exacto de pacientes que logra tener un estudiante en cada una de sus rotaciones por los sitios de práctica, el marco muestral se realizó por reposición de datos con una confiabilidad del 95\% y un margen de error de $5 \%$, lo que generó una muestra total de 356 personas encuestadas.

\section{Criterio de inclusión}

Pacientes que estuvieron al cuidado del estudiante mínimo 12 horas o en un turno de 7 am a 7 pm.

\section{Criterios de exclusión}

Pacientes con alguna alteración cognitiva o psicológica para responder el cuestionario; para el caso específico del hospital infantil, por ser menores de edad, se aplicó el cuestionario a su cuidador principal.

\section{Técnicas e instrumentos de recolección de información}

Se utilizó el instrumento denominado "Percepción de Comportamientos de Cuidado Humanizado de Enfermería (PCHE) Versión 3", validado González en el 2013(9), se trata de una encuesta autodirigida, de 32 ítems con una escala Likert de 4 opciones en donde se asigna un valor a cada pregunta así: nunca $=1$ punto, algunas veces $=2$ puntos, casi siempre $=3$ puntos $\mathrm{y}$ siempre $=4$ puntos, tiene un puntaje mínimo de $42 \mathrm{y}$ máximo de 168 puntos. La fiabilidad de los ítems del cuestionario aplicado reporta un Alfa de Cronbach de 0,877 . Los ítems se enuncian de manera positiva y están distribuidos de manera interna en 3 categorías que son: priorizar el sujeto de cuidado (la persona), apertura a la comunicación para proporcionar educación en salud a la persona y cualidades del hacer de enfermería.

El cuestionario utilizado permite ubicar la percepción del cuidado humanizado de la siguiente forma: siempre se percibe una relación de cuidado humanizado de enfermería (puntuación de la escala de 104,1 -128). Casi siempre se percibe el cuidado de enfermería (puntuación de la escala entre 80,1 - 104). Algunas veces se percibe el cuidado de enfermería (puntuación de la escala entre 56,1 - 80). Ninguna Percepción del cuidado (puntuación de la escala entre 32 - 56). Cada entrevista tuvo una duración aproximada de 20 a 25 minutos por persona.

\section{Procesamiento y análisis de la información}

Para la operacionalización de la información se creó una matriz en Microsoft Excel y posterior procesamiento en el Programa Estadístico para las Ciencias Sociales (IBM SPSS v. 22). Para el análisis se utilizó la estadística descriptiva mediante frecuencias, medidas de tendencia central, tablas de contingencia y análisis de significancia con la prueba no paramétrica de Kruskall-Wallis considerando un $\alpha=0,01$.

\section{Consideraciones éticas}

La presente investigación se catalogó con riesgo mínimo según Resolución 008430 de 1993, ya que no se realizó ninguna intervención o modificación intencionada de las variables biológicas, fisiológicas, sicológicas o sociales de las personas que 
participaron en el estudio. Para la recolección de la información se obtuvo autorización de los comités de ética y/o investigaciones de cada institución y el consentimiento informado de cada uno de los participantes.

\section{Resultados}

El total de la muestra recolectada fue de 356 pacientes distribuidos por cada institución de la siguiente manera: Hospital infantil 78 pacientes (21,9\%), Hospital San Marcos de Chinchiná 72 pacientes $(20,2 \%)$, Clínica Versalles 72 pacientes (20,2\%), Assbasalud Enea 60 pacientes (16,8\%), Assbasalud Cayetano 48 pacientes $(13,4 \%)$ e Instituto DIACORSA 26 pacientes $(7,3 \%)$.

\section{Cuidado humanizado global}

En el análisis del cuestionario se obtuvo un dato mínimo de 72 puntos y un dato máximo de 128 puntos, con un promedio de 120 puntos para toda la muestra. En este sentido, se interpreta de acuerdo al instrumento, que la población de personas atendidas por los estudiantes de enfermería considera que "siempre se percibe una relación de cuidado humanizado".

\section{Cuidado humanizado por categorías}

En la primera categoría, priorizar el sujeto de cuidado (la persona) se encontró que el $88,3 \%$ de los pacientes respondieron siempre y $9,9 \%$ casi siempre; para la categoría de apertura a la comunicación, para proporcionar educación en salud a la persona, el 76,5\% de los pacientes respondieron siempre, $15,3 \%$ casi siempre, por último en la categoría cualidades del hacer de enfermería, se encontró que el $82,9 \%$ respondió siempre, $11,6 \%$ casi siempre (Figura 1).

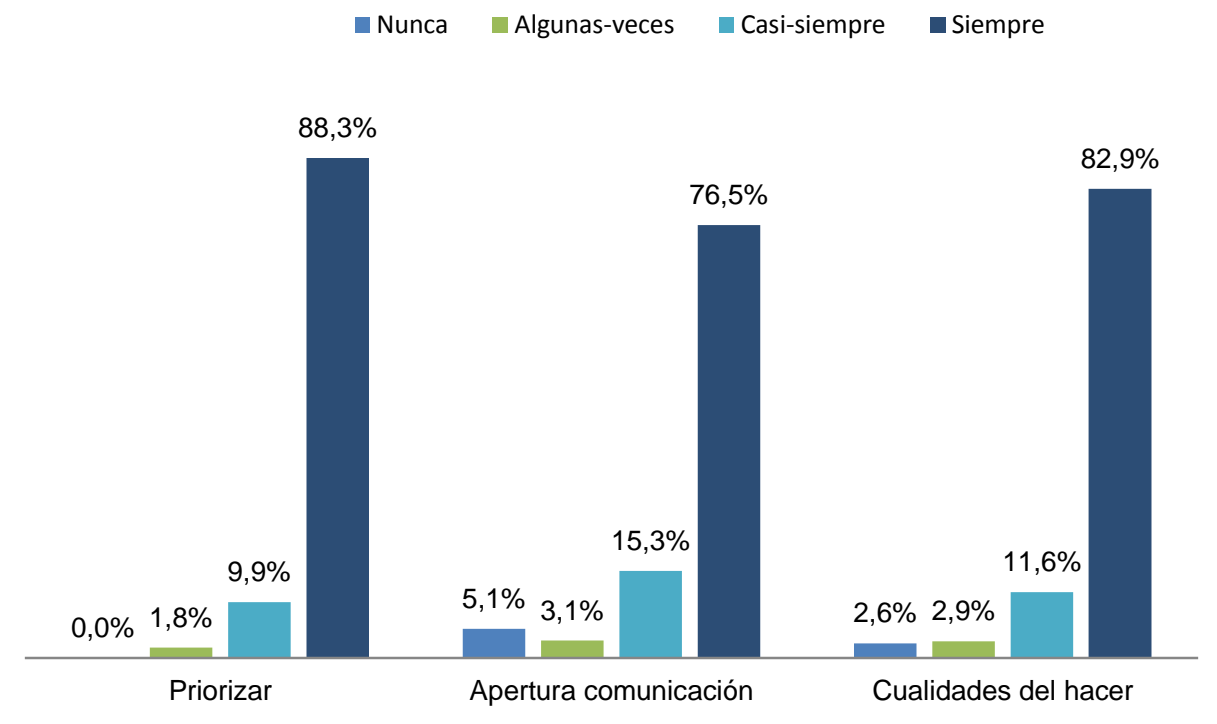

Figura 1. Resultado de la percepción por categorías del paciente hospitalizado sobre cuidado humanizado de enfermeros de la Universidad Católica de Manizales

\section{Análisis por categorías de cuidado}

Al realizar el análisis de las preguntas que contiene la categoría priorizar el sujeto de cuidado, se evidencian respuestas con alto porcentaje en "siempre"; sin embargo, es importante prestar atención a las siguientes respuestas: "Le demuestran interés por sus creencias y le hacen sentirse tranquilo cuando están con usted", con valores de casi siempre $15 \%$ y siempre $82 \%$ respectivamente; y "Le generan confianza cuando lo cuidan" con valores de casi siempre $14 \%$ y siempre $83 \%$. (Figura 2). 
1. Le hacen sentir como una persona

2. Le tratan con amabilidad

15. Le explican los cuidados usando un tono de voz pausado

6. Le hacen sentirse bien atendido cuando dialogan con usted

8. Le generan confianza cuando lo(la) cuidan

17. Le demuestran respeto por sus creencias y valores

7. El personal de enfermería le hace sentirse tranquilo(a), cuando está con usted

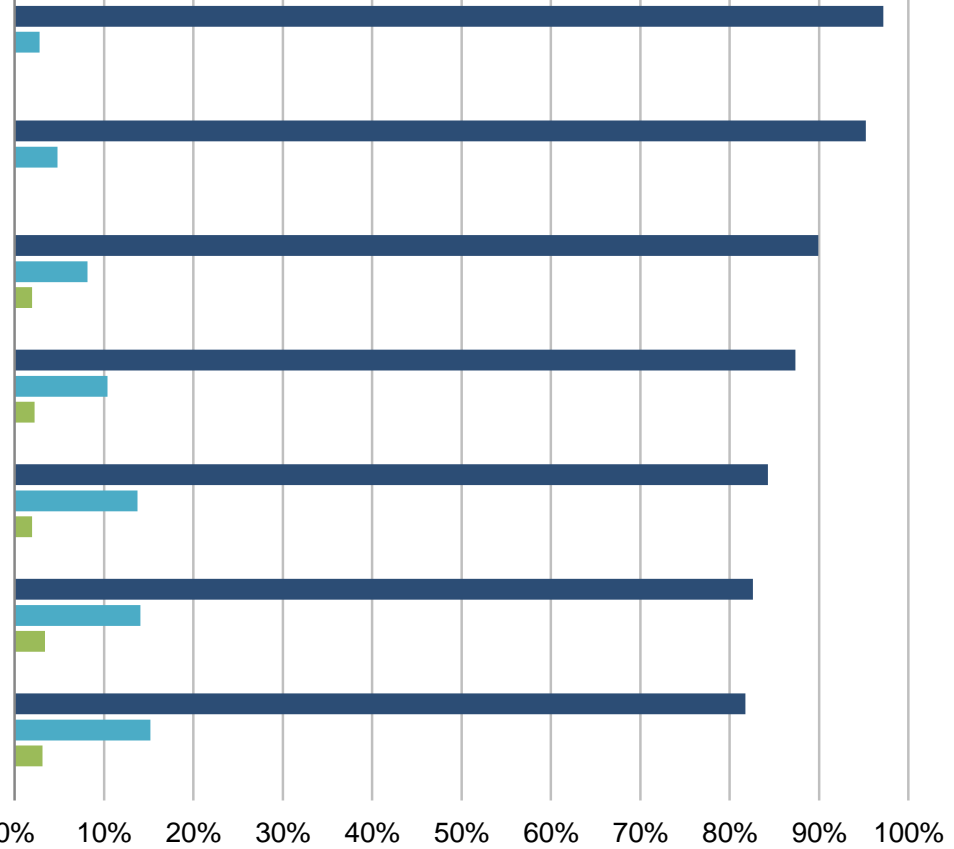

- Siempre $\quad$ Casi-siempre $\square$ Algunas-veces $\square$ Nunca

Figura 2. Priorizar el sujeto de cuidado: la persona

Dentro de la categoría de apertura a la comunicación para proporcionar educación en salud a la persona, se evidenció variación de las respuestas de los encuestados, en el ítem de: "Le dan indicaciones sobre su autocuidado", con un resultado de siempre $65 \%$, casi siempre $11 \%$, y nunca $20 \%$; el ítem "Le indican su nombre y cargo antes de realizarle los procedimientos", mostró variabilidad presentando un $12 \%$ de nunca (Figura 3 ).

5. Le dedican tiempo para aclararle sus inquietudes

4. Le miran a los ojos, cuando le hablan

10. Le explican previamente los procedimientos

12. Le indican su nombre y cargo antes de realizarle los procedimientos

9. Le facilitan el diálogo

11. Le responden con seguridad y claridad a sus preguntas

19. Le proporcionan información suficiente y oportuna para que pueda tomar decisiones sobre su situación de salud

14. Le dan indicaciones sobre su autocuidado -los cuidados que usted debe seguir-, de manera oportuna

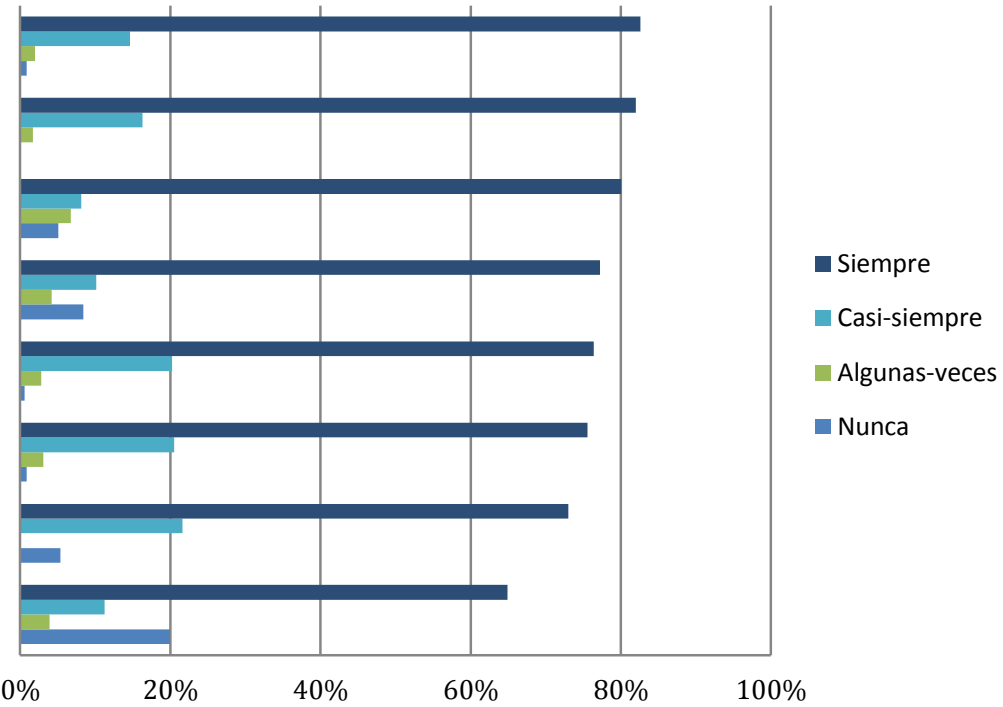

Figura 3. Apertura a la comunicación para proporcionar educación en salud a la persona 
Con respecto a la categoría de cualidades del hacer de enfermería se presentó variabilidad de respuestas a las preguntas: "Le indican sus necesidades de tipo físico, psicológico y espiritual”, donde el 64\% expresó siempre, $16,9 \%$ casi siempre, $8,1 \%$ algunas veces $\mathrm{y}$ nunca 12,6\%; de igual manera en: "Le preguntan y se preocupan pos su estado de ánimo", evidenció un resultado de siempre $73,2 \%$, casi siempre $12,1 \%$, algunas veces $4,4 \%$ y nunca $9,3 \%$ y en el ítem: " $L e$ permiten expresar sus sentimientos sobre la enfermedad y tratamiento" con 72,8\%, siempre, $18,3 \%$ casi siempre, $4,8 \%$ algunas veces y nunca de $4,2 \%$. En el resto de las preguntas se presentó respuestas principalmente en las categorías de siempre y casi siempre. (Figura 4).
31. Le respetan su intimidad

3. Le muestran interés por brindarle comodidad durante su hospitalización

29. Le respetan sus decisiones

32. Le administran a tiempo los medicamentos formulados por el médico

26. Le brindan un cuidado cálido y delicado

28. Le demuestran que son responsables con su atención

16. Le llaman por su nombre

27. Le ayudan a manejar su dolor físico

24. Le escuchan atentamente

18. Le atienden oportunamente sus necesidades básicas (higiene, alimentación, evacuación urinaria e intestinal)

20. Le manifiestan que están pendientes de usted

13. Le dedican el tiempo requerido para su atención

30. Le indican que cuando requiera algo, usted les puede llamar

22. Responden oportunamente a su llamado

25. Le preguntan y se preocupan por su estado de ánimo

21. Le permiten expresar sus sentimientos sobre la enfermedad y tratamiento

23. Identifican sus necesidades de tipo físico, psicológico y espiritual

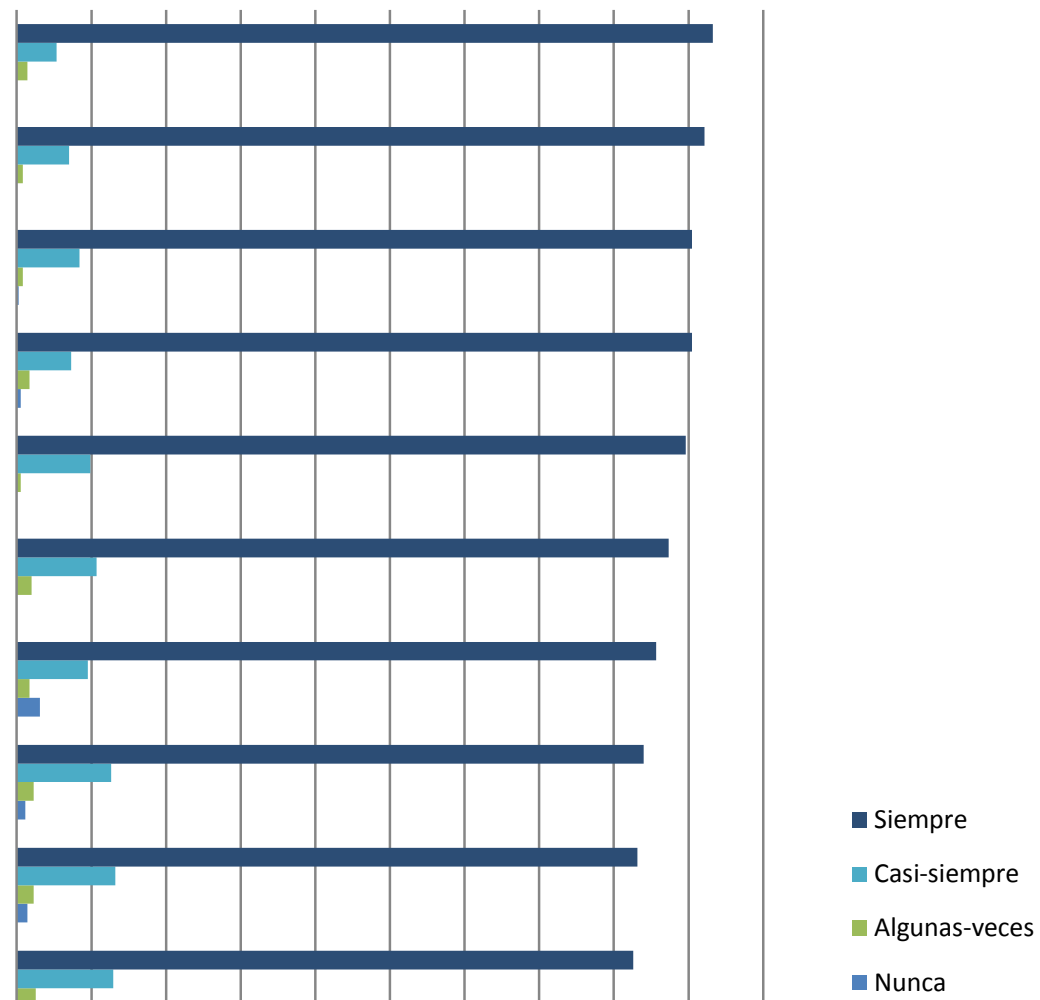

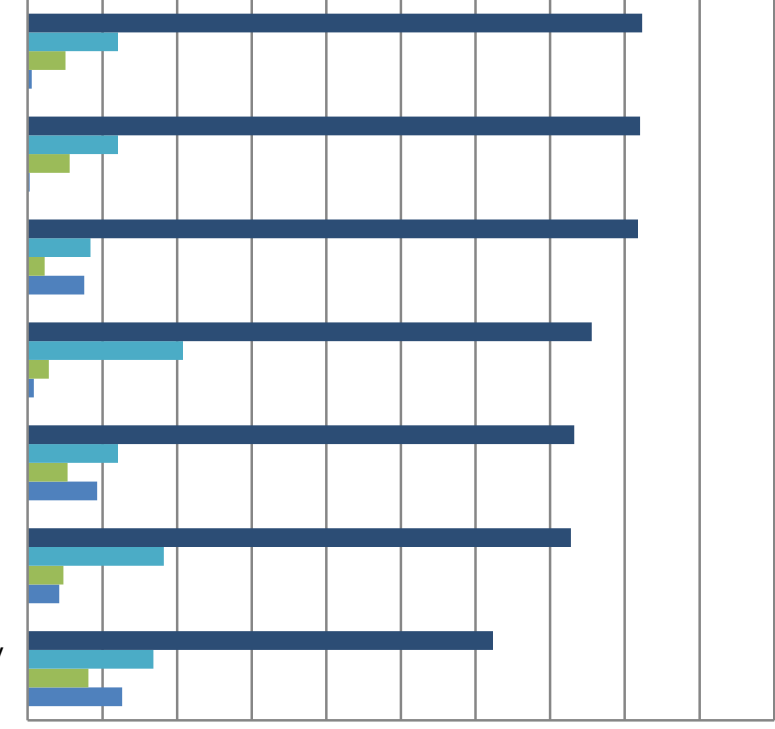

$\begin{array}{llllllllllll}0 \% & 10 \% & 20 \% & 30 \% & 40 \% & 50 \% & 60 \% & 70 \% & 80 \% & 90 & 100 \%\end{array}$

Figura 4. Cualidades del hacer de enfermería 
En el análisis de Kruskall-Wallis se comparó el puntaje global con cada pregunta de la categoría priorizar el sujeto de cuidado (la persona) y se encontraron relaciones estadísticamente significativas en las preguntas: "el personal de enfermería le hace sentirse tranquilo cuando está con usted"; "le hacen sentirse bien atendido cuando dialogan con usted"; "le generan confianza cuando lo cuidan"; "le explican los cuidados usando un tono de voz pausado"; "le demuestran respeto por sus creencias y valores" (Tabla 1 ).

Tabla 1. Análisis descriptivo de la priorización del sujeto de cuidado

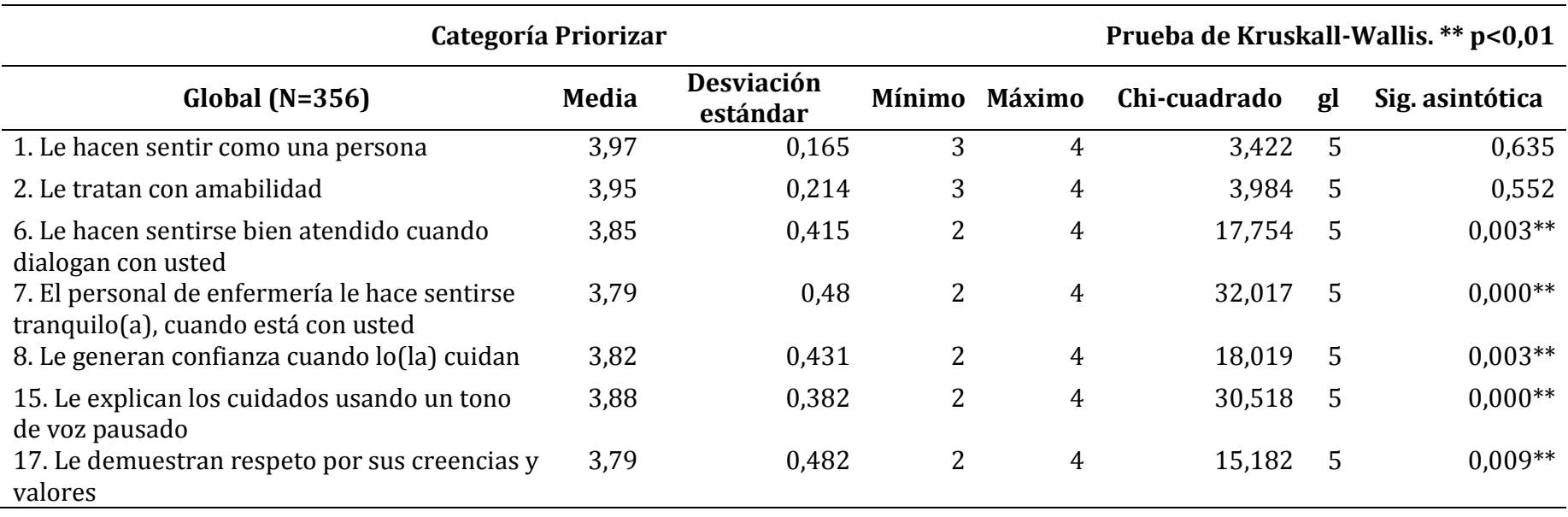

En cuanto a la categoría apertura a la comunicación donde se compara con el puntaje global, se encontraron relaciones estadísticamente significativas en todas las preguntas (Tabla 2). Para la categoría cualidades del hacer de enfermería, se encontraron relaciones estadísticamente significativas en todas las preguntas, excepto en las preguntas: "le muestran interés por brindarle comodidad durante su hospitalización" y "le escuchan atentamente" (Tabla 3).

Tabla 2. Análisis descriptivo de la apertura a la comunicación

\begin{tabular}{|c|c|c|c|c|c|c|c|}
\hline \multicolumn{4}{|c|}{ Categoría Apertura a la comunicación } & \multicolumn{4}{|c|}{ Prueba de Kruskall-Wallis. ${ }^{* *} \mathbf{p}<0,01$} \\
\hline Global $(\mathrm{N}=356)$ & Media & $\begin{array}{l}\text { Desviación } \\
\text { estándar }\end{array}$ & Mínimo & Máximo & Chi-cuadrado & gl & Sig. asintótica \\
\hline 4. Le miran a los ojos, cuando le hablan & 3,8 & 0,44 & 2 & 4 & 30,398 & 5 & $0,00001^{* *}$ \\
\hline $\begin{array}{l}\text { 5. Le dedican tiempo para aclararle sus } \\
\text { inquietudes }\end{array}$ & 3,79 & 0,51 & 1 & 4 & 32,693 & 5 & $0,0000^{* *}$ \\
\hline 9. Le facilitan el diálogo & 3,72 & 0,54 & 1 & 4 & 18,553 & 5 & $0,002^{* *}$ \\
\hline $\begin{array}{l}\text { 10. Le explican previamente los } \\
\text { procedimientos }\end{array}$ & 3,63 & 0,82 & 1 & 4 & 38,607 & 5 & $0,0000^{* *}$ \\
\hline $\begin{array}{l}\text { 11. Le responden con seguridad y claridad } \\
\text { a sus preguntas }\end{array}$ & 3,71 & 0,57 & 1 & 4 & 29,307 & 5 & $0,00002^{* *}$ \\
\hline $\begin{array}{l}\text { 12. Le indican su nombre y cargo antes de } \\
\text { realizarle los procedimientos }\end{array}$ & 3,56 & 0,92 & 1 & 4 & 38,843 & 5 & $0,0000^{* *}$ \\
\hline $\begin{array}{l}\text { 14. Le dan indicaciones sobre su } \\
\text { autocuidado -los cuidados que usted debe } \\
\text { seguir-, de manera oportuna }\end{array}$ & 3,21 & 1,2 & 1 & 4 & 89,787 & 5 & $0,0000^{* *}$ \\
\hline $\begin{array}{l}\text { 19. Le proporcionan información } \\
\text { suficiente y oportuna para que pueda } \\
\text { tomar decisiones sobre su situación de } \\
\text { salud }\end{array}$ & 3,62 & 0,75 & 1 & 4 & 34,014 & 5 & $0,0000^{* *}$ \\
\hline
\end{tabular}


Tabla 3. Análisis descriptivo de las cualidades del hacer de enfermería

\begin{tabular}{|c|c|c|c|c|c|c|c|}
\hline \multicolumn{5}{|c|}{ Categoría Cualidades del hacer de enfermería } & \multicolumn{3}{|c|}{ Prueba de Kruskall-Wallis. ${ }^{* *} \mathbf{p}<0,01$} \\
\hline Global $(N=356)$ & Media & $\begin{array}{l}\text { Desviación } \\
\text { estándar }\end{array}$ & Mínimo & Máximo & Chi-cuadrado & gl & Sig. asintótica \\
\hline $\begin{array}{l}\text { 3. Le muestran interés por brindarle } \\
\text { comodidad durante su hospitalización }\end{array}$ & 3,91 & 0,31 & 2 & 4 & 13,908 & 5 & 0,116 \\
\hline $\begin{array}{l}\text { 13. Le dedican el tiempo requerido para su } \\
\text { atención }\end{array}$ & 3,76 & 0,56 & 1 & 4 & 17,138 & 5 & $0,0042^{* *}$ \\
\hline 16. Le llaman por su nombre & 3,78 & 0,63 & 1 & 4 & 53,751 & 5 & $0,0000^{* *}$ \\
\hline $\begin{array}{l}\text { 18. Le atienden oportunamente sus } \\
\text { necesidades básicas } \\
\text { alimentación, evacuación urinaria ene, } \\
\text { intestinal) }\end{array}$ & 3,76 & 0,59 & 1 & 4 & 13,641 & 5 & $0,0181^{*}$ \\
\hline $\begin{array}{l}\text { 20. Le manifiestan que están pendientes de } \\
\text { usted }\end{array}$ & 3,76 & 0,56 & 1 & 4 & 16,59 & 5 & $0,0053^{* *}$ \\
\hline $\begin{array}{l}\text { 21. Le permiten expresar sus sentimientos } \\
\text { sobre la enfermedad y tratamiento }\end{array}$ & 3,6 & 0,77 & 1 & 4 & 55,286 & 5 & $0,0000^{* *}$ \\
\hline $\begin{array}{l}\text { 22. Responden oportunamente a su } \\
\text { llamado }\end{array}$ & 3,71 & 0,56 & 1 & 4 & 33,545 & 5 & $0,0000^{* *}$ \\
\hline $\begin{array}{l}\text { 23. Identifican sus necesidades de tipo } \\
\text { físico, psicológico y espiritual }\end{array}$ & 3,29 & 1,06 & 1 & 4 & 97,326 & 5 & $0,0000^{* *}$ \\
\hline 24. Le escuchan atentamente & 3,78 & 0,55 & 1 & 4 & 5,883 & 5 & 0,3178 \\
\hline $\begin{array}{l}\text { 25. Le preguntan y se preocupan por su } \\
\text { estado de ánimo }\end{array}$ & 3,49 & 0,96 & 1 & 4 & 77,953 & 5 & $0,0000^{* *}$ \\
\hline 26. Le brindan un cuidado cálido y delicado & 3,89 & 0,33 & 2 & 4 & 17,037 & 5 & $0,0000^{* *}$ \\
\hline 27. Le ayudan a manejar su dolor físico & 3,79 & 0,53 & 1 & 4 & 24,924 & 5 & $0,0001^{* *}$ \\
\hline $\begin{array}{l}\text { 28. Le demuestran que son responsables } \\
\text { con su atención }\end{array}$ & 3,85 & 0,41 & 2 & 4 & 19,268 & 5 & $0,0017^{* *}$ \\
\hline 29. Le respetan sus decisiones & 3,89 & 0,36 & 1 & 4 & 20,998 & 5 & $0,0008^{* *}$ \\
\hline $\begin{array}{l}\text { 30. Le indican que cuando requiera algo, } \\
\text { usted les puede llamar }\end{array}$ & 3,64 & 0,86 & 1 & 4 & 31,896 & 5 & $0,0000^{* *}$ \\
\hline 31. Le respetan su intimidad & 3,92 & 0,32 & 2 & 4 & 18,199 & 5 & $0,0027^{* *}$ \\
\hline $\begin{array}{l}\text { 32. Le administran a tiempo los } \\
\text { medicamentos formulados por el médico }\end{array}$ & 3,88 & 0,42 & 1 & 4 & 22,503 & 5 & $0,0004^{* *}$ \\
\hline
\end{tabular}

\section{Discusión}

El presente estudio evidenció que de manera general loa pacientes siempre perciben cuidado humanizado de los estudiantes de enfermería, similar a estudios referenciados que evaluaron la percepción del cuidado humanizado para el caso de profesionales de enfermería, en donde además se hace énfasis en la labor del personal desde la empatía, adecuada comunicación y actitud(10-14). La gran mayoría de investigaciones que miden la percepción del paciente a cerca del cuidado ofrecido se fundamenta en profesionales de enfermería más que en estudiantes, solo se documentó el estudio de González, que realizó su estudio con estudiantes de enfermería de la Universidad Francisco de Paula Santander, utilizando el instrumento PCHE Versión 2, y presentó resultados de percepción de cuidado humanizado relativamente bajo ya que el 60,7\% manifestó que la percibía "en algunas ocasiones", 17,9\% "nunca" y solo el 21,4\% "siempre"(15).

Guerrero, et al., realizaron en el 2015, medición del cuidado humanizado brindado por enfermeras a través de la escala de medición de cuidado humano de Watson y concluyeron que este era brindado de forma regular y por ello se sugirió implementar estrategias, planes de mejora y capacitación continua con la finalidad de generar la sensibilización del personal de enfermería para ofrecer buen trato al paciente desde un abordaje basado en valores humanos(16).

Se han encontrado estudios relacionados con medición de la satisfacción del paciente y con la valoración de cuidado humanizado, así como instrumentos para medir el cuidado humanizado desde diferentes perspectivas como: la valoración del cuidado humanizado, percepción y/o habilidades para el cuidado; todos ellos enfocados a la búsqueda 
del mejoramiento permanente de los procesos de atención, cuidado directo y fortalecimiento de la praxis desde los sustentos teóricos de la disciplina de enfermería(5-10,17-19).

Es posible que exista una relación entre los procesos formativos centrados en una educación integral con el cuidado humanizado que brindan a los pacientes, como es el caso de los estudiantes involucrados en la presente investigación, en donde desde la educación con personalización liberadora se fomenta la formación integral desde una visión humanista, científica y cristiana; evidencia de esta asociación la presentan estudios enfocados en los estudiantes, sus experiencias en prácticas y capacidades, demostrando habilidades para el cuidado humanizado, paciencia, y conocimiento, que pueden ser tomados como referentes fundamentales de los proyectos educativos de los programas de enfermería para fortalecer sus currículos $(2,17,20)$.

Siappo et al.(20) expresan que el modelo de formación centrado en una cultura estrictamente profesional y técnica no está contribuyendo para la formación de ciudadanos críticos-reflexivos y por ello es relevante superar este modelo con una perspectiva ética y humanística. González ${ }^{(21)}$ por su parte, expresa que el marco de la relación docente-estudiante existente, se pone en evidencia el papel que tiene la educación en el desarrollo de actitudes, conductas y valores, incluidos aspectos técnico-científicos, interpersonales, afectivos y éticos relacionados con el cuidado. Ferro, et al. estudiaron los significados sobre el "arte del cuidar" desde la perspectiva de los estudiantes de enfermería y destacan que la formación humanística se centra en cualificar al cuidado como holístico, con extensión a la familia, donde se reconocen los sentimientos implicados; hacen énfasis en el desarrollo de la identidad profesional-personal, como componente esencial de la enfermería(22).

En este sentido se puede interpretar que en este estudio la persona percibe favorablemente el cuidado humanizado por parte del estudiante, dado ellos propician un acompañamiento directo e integral; mientras que, en el ámbito laboral, en los enfermeros profesionales predomina la visión biomédica, la supremacía técnica y la fragmentación de la persona, lo que ocasiona un contexto que favorece la deshumanización de los cuidados de enfermería(7).
Es importante resaltar el estudio fenomenológico realizado por Beltrán(23), en donde indaga experiencias de cuidado humanizado y muestra como la receptividad sin discriminación, el interés por cuidar, escuchar las quejas, cuidar en todas las esferas e involucrar a la familia, son detalles imprescindibles en el cuidado humanizado. Elementos fundamentales a tener en cuenta en las áreas donde el futuro profesional tiene mayor contacto directo con el paciente; es así que los comportamientos de cuidado difieren de los modelos que guíen la formación práctica y las oportunidades basadas en el cúmulo de conocimiento y destreza profesional, es de vital importancia el comportamiento de cuidado de las enfermeras asistenciales y docentes en la preparación de los estudiantes para el desarrollo de las prácticas clínicas(11).

\section{Conclusiones}

Conocer la percepción que tiene la persona, de cómo se siente al cuidado de un futuro profesional de enfermería, muestra el impacto de las acciones de cuidado y pone en evidencia la necesidad de fortalecer aspectos específicos como la comunicación y abordar las necesidades personales, espirituales y de autocuidado de la persona; resultados que a pesar de no haber sido evaluados de manera negativa, deben ser revisados minuciosamente para mejorar el acto del cuidado y repensar en la disciplina su praxis, así como el impacto generado entre la teoría y la práctica para recuperar los aspectos perdidos e invisibles de la enfermería.

Las instituciones de formación de profesionales de enfermería requieren una autoevaluación permanente a los contenidos curriculares, su coherencia y pertinencia para la formación del capital humano con sentido social. Es necesario dar un valor que legitime el cuidado humano como sello particular de la academia desde las diferentes dimensiones del ser. Para propiciar el actuar enfermero, en el acto formativo debe existir una conexión fundamental entre los perfiles de formación y la investigación, generando construcción de conocimientos derivados de una problemática propia de enfermería.

Lograr que al momento de interacción enfermero persona, se establezca un vínculo que va más allá del simple hecho de brindar atención con calidad, como compromiso inherente al que hacer profesional, es posible cuando las competencias del ser y el hacer, 
posibilitan el cuidado humanizado, dentro de las dimensiones físicas, espirituales e individuales de la persona, y es ese momento en el que el profesional de enfermería reconoce que está en la capacidad de desarrollar lo aprendido dentro de sus procesos formativos enmarcados dentro los objetivos de formación de las escuelas de enfermería, sus referentes teóricos y modelos pedagógicos que vislumbran un sello particular en cada estudiante el proceso de formación.

\section{Recomendaciones}

Es necesario que los programas y facultades de enfermería y en general de ciencias de la salud realicen un análisis profundo a la forma como se visualiza el quehacer de los estudiantes en las practica formativa, en donde efectivamente hacen realidad sus experiencias de cuidado y contacto con el paciente y pueden hacer uso de las estrategias, conocimientos teóricos y habilidades que se considera debería haber adquirido; de ésta manera realmente se consigue comprender las dimensiones más profundas del currículo y con base en resultados reales se puede plantear estrategias de mejoramiento y seguimiento al proceso de formación del futuro profesional.

\section{Conflicto de intereses}

Ninguno declarado por los autores.

\section{Referencias}

1. Borré-Ortiz YM, Lenis-Victoria C, Suárez-Villa M, TafurCastillo J. El conocimiento disciplinar en el currículo de enfermería: una necesidad vital para transformar la práctica. Revista Ciencias de la Salud [Internet]. 2015;13(3):481-491. Disponible https://www.redalyc.org/articulo.oa?id=56242524013. http://dx.doi.org/10.12804/revsalud13.03.2015.12

2. Valderrama-Sanabria ML, Peña-Pita AP, Clavijo-Álvarez LM. Narrativa: El estudiante de enfermería aprendiendo el arte de cuidar. Rev Cuid [Internet]. 2017 [citado 23 abr 2019]; 8(1):1488-1498. Disponible

en: http://www.scielo.org.co/scielo.php?script=sci_arttext\&pid =S2216-09732017000101488\&lng=en. http://dx.doi.org/10.15649/cuidarte.v8i1.362.

3. Balderas-Pedreros M. Administración de los servicios de enfermería. 7a ed. México: Mc; 2015. 301 p.

4. Gualdrón M, Barrera-Ortega K, Parra-González N. Percepción de la enseñanza en cuidado humanizado vs la práctica formativa por estudiantes de enfermería. Aibi Revista De Investigación, Administración E Ingeniería [Internet]. 2019. [citado 17 abr 2019]; 7(1):7-12. Disponible en: https://revistas.udes.edu.co/aibi/article/view/507

5. Morales-Castillo FA, Hernández-Cruz MC, Morales-Rodríguez MC, Landeros-Olvera EA. Validación y estandarización del instrumento: Evaluación de los comportamientos de cuidado otorgado en enfermeras mexicanas. Enferm. univ [Internet]. 2016 Mar [citado 17 abr 2019]; 13(1): 3-11. Disponible en: http://www.scielo.org.mx/scielo.php?script=sci_arttext\&pi $\mathrm{d}=\mathrm{S} 1665-70632016000100003 \& \operatorname{lng}=\mathrm{es}$.

http://dx.doi.org/10.1016/j.reu.2015.11.005.

6. Romero-Massa E, Contreras-Méndez IM, Moncada-Serrano A. Relación entre cuidado humanizado por enfermería con la hospitalización de pacientes. Hacia promoc. Salud [Internet]. 2016 June [citado 17 abr 2019]; 21(1):26-36. Disponible en: http://www.scielo.org.co/scielo.php?script=sci_arttext\&pid =S0121-75772016000100003\&lng=en.

http://dx.doi.org/10.17151/hpsal.2016.21.1.3

7. Salgado J, Valenzuela S, Saez K. Comportamientos del cuidado percibidos por estudiantes de enfermería y receptores del cuidado. Cienc. enferm. [Internet]. 2015 Abr [citado 17 abr 2019]; 21(1): 69-79. Disponible en: https://scielo.conicyt.cl/scielo.php?script=sci_arttext\&pid= S0717-95532015000100007\&lng=es.

http://dx.doi.org/10.4067/S0717-95532015000100007

8. Izquierdo-Machín E. Enfermería: Teoría de Jean Watson y la inteligencia emocional, una visión humana. Rev Cubana Enfermer [Internet]. 2015 Sep [citado 17 abr 2019]; 31(3). Disponible

http://scielo.sld.cu/scielo.php?script=sci_arttext\&pid=S086 4-03192015000300006\&lng=es.

9. González Hernández Oscar Javier. Validez y confiabilidad del instrumento "Percepción de comportamientos de cuidado humanizado de enfermería PCHE $3^{a}$ versión". Aquichan [Internet]. 2015 July [citado 17 abr 2019] ; 15(3): 381-392. Disponible en: http://www.scielo.org.co/scielo.php?script=sci_arttext\&pid $=$ S1657-59972015000300006\&lng=en. http://dx.doi.org/10.5294/aqui.2015.15.3.6

10. Herrera-Zuleta I, Bautista-Perdomo L, López-Reina M, Ordoñez-Correa M, Rojas-Rivera J, Suarez-Riascos H, VallejoMoreno J. Percepciones de las gestantes en torno al cuidado humanizado por enfermería. Rev. cienc. cuidad. [Internet]. 30 dic. 2016 [citado 23 abr.2019];13(2):58-2. Disponible en: https://revistas.ufps.edu.co/index.php/cienciaycuidado/art icle/view/763

11. Bautista-Rodríguez LM, Arias-Velandia MF, Carreño-Leiva ZO. Percepción de los familiares de pacientes críticos hospitalizados respecto a la comunicación y apoyo emocional. Rev Cuid [Internet]. 2016 [citado 17 abr 2019]; 7(2):1297-1309. Disponible en: http://www.scielo.org.co/scielo.php?script=sci_arttext\&pid =S2216-09732016000200007\&lng=en. http://dx.doi.org/10.15649/cuidarte.v7i2.330.

12. Ventocilla-Martínez F. Percepción del paciente sobre el cuidado humanizado que brinda el profesional de enfermería en el servicio de observación del Hospital de Emergencia Casimiro Ulloa 2015. Trabajo de investigación. Perú: Universidad Nacional de Mayor de San Marcos; 2017. Disponible

en: http://cybertesis.unmsm.edu.pe/bitstream/handle/cyberte sis/6302/Ventocilla_mf.pdf?sequence $=1$

13. Monje P, Miranda P, Oyarzün J, Seguel Fredy, Flores E. Percepción de cuidado humanizado de enfermería desde la perspectiva de usuarios hospitalizados. Cienc. enferm. [Internet]. 2018 [citado 21 abr 2019]; 24:5. Disponible en: https://scielo.conicyt.cl/scielo.php?script=sci_arttext\&pid= S0717-95532018000100205\&lng=es. Epub 25-Sep-2018. http://dx.doi.org/10.4067/s0717-95532018000100205. 
14. Bautista-Rodríguez LM, Parra-Carrillo EL, Arias-Torres KM, Parada-Ortiz KJ, Ascanio-Meza KA, Villamarin-Capacho MI, et al. Percepción de los comportamientos de cuidado humanizado en los usuarios hospitalizados en una institución de salud de $3^{\circ}$ y $4^{\circ}$ nivel de atención. Rev. cienc. cuidad. [Internet]. 2015; [citado 21 abr 2019] 12(1):105-118. Disponible en: https://dialnet.unirioja.es/servlet/articulo?codigo $=524398$ 8

15. González-Escobar D. Percepción de comportamientos de cuidado humanizado en estudiantes de enfermería. Rev actual enf. [Internet]. 2014 [citado 2019 Abr 17] 17(2) Disponible en: https://encolombia.com/medicina/revistasmedicas/enfermeria/ve-172/percepcion-comportamientosmateriales/

16. Guerrero-Ramírez R, Meneses-La Riva ME, De La Cruz-Ruiz M. Cuidado humanizado de enfermería según la teoría de Jean Watson, servicio de medicina del Hospital Daniel Alcides Carrión. Lima- Callao, 2015. Rev enferm Herediana. [Internet]. 2016; [citado 17 abr 2019]; 9(2):133-142. Disponible en: http://www.upch.edu.pe/vrinve/dugic/revistas/index.php /RENH/article/viewFile/3017/2967

17. Landman-Navarro A, del Alcazar-Pabst R, Madrid-Zamorano Y, Pais Veliz L, Rosenkranz-Moreno E, Vivanco-Lobato I. Habilidades para el cuidado humanizado de estudiantes de enfermería. Enfermería: Cuidados humanizados. [Internet]. 2016 Jul [citado 23 abr 2019]; 5(1):29-34. Disponible en: <http://revistas.ucu.edu.uy/index.php/enfermeriacuidados humanizados/article/view/11

18. Rodríguez Angélica Melita, Concha Patricia Jara, Pereira Daniel Ignacio, Machuca Luis Luengo. Adaptación transcultural y validación de un cuestionario de cuidado humanizado en enfermería para una muestra de población Chilena. Rev Cuid [Internet]. 2018 [citado 2019 Abr 21]; 9(2):2245-2256. Disponible en: http://www.scielo.org.co/scielo.php?script=sci_arttext\&pid $=$ S2216-
19. Hermosilla-Ávila A, Mendoza-Llanos R, Contreras-Contreras S. Instrumento para valoración del cuidado humanizado brindado por profesionales de enfermería a personas hospitalizadas. Index Enferm [Internet]. 2016 Dic [citado 2019 Abr 21]; 25(4):273-277. Disponible en: http://scielo.isciii.es/scielo.php?script=sci_arttext\&pid=S11 32-12962016000300011\&lng=es.

20. Gorriti-Siappo CL, Rodríguez-Núñez Y, Evangelista-Cabral I. Experiencias de estudiantes de enfermería en el cuidado de si durante el proceso de formación en una universidad privada en Chimbote, Perú. Esc. Anna Nery [Internet]. 2016 Mar [citado 23 abr 2019]; 20(1):17-24. Disponible en: http://www.scielo.br/scielo.php?script=sci_arttext\&pid=S1 414-81452016000100017\&lng=en. http://dx.doi.org/10.5935/1414-8145.20160003.

21. González-Escobar DS. Significado del cuidado para estudiantes y profesores del programa de enfermería de la Universidad Francisco de Paula Santander. Investigación en Enfermería: Imagen y Desarrollo [Internet]. 2015 [citado 23 abr 2019]; 17(1):77-95. Disponible en: https://www.redalyc.org/articulo.oa?id=145233516006

22. Ferro-Rivera N, Rodríguez-Jiménez S, Cárdenas-Jiménez M. Significados sobre a arte do cuidado desde a perspectiva dos estudiantes de Licenciatura em Enfermagem. Enferm. univ [revista en la Internet]. 2018 Dic [citado 2019 Abr 21]; 15(4):402-415. Disponible en: http://www.scielo.org.mx/scielo.php?script=sci_arttext\&pi $\mathrm{d}=\mathrm{S} 1665-70632018000400402 \& \operatorname{lng}=\mathrm{es}$. http://dx.doi.org/10.22201/eneo.23958421e.2018.4.541.

23. Beltrán-Salazar Ó. Atención al detalle, un requisito para el cuidado humanizado. Index Enferm [Internet]. 2015 Jun [citado 2019 Abr 17]; 24(1-2):49-53. Disponible en: http://scielo.isciii.es/scielo.php?script=sci_arttext\&pid=S11 32-12962015000100011\&lng=es. http://dx.doi.org/10.4321/S1132$1296201500010001109732018000202245 \& \operatorname{lng}=e s$. http://dx.doi.org/10.15649/cuidarte.v9i2.531. 